SHS Web of Conferences 7, 01008 (2014)

DOI: $10.1051 /$ shsconf / 20140701008

C) Owned by the authors, published by EDP Sciences, 2014

\title{
Research and Application of Birth-death of Equipment in Overhaul Plan for Distribution Grid
}

\author{
Liu Xusheng \\ Info-Communication Company, Xinzhou Power Supply Company of Shanxi Power Company, 034000 \\ Xinzhou Shanxi, China
}

\begin{abstract}
Overhaul of distribution grid plays a decisive role in the operation and dispatch of power grid. With the increasingly expansion of power grid scale, its structure tends to be complicated as well. The traditional overhaul plan mode is currently not able to meet the development demand of power grid enterprises. Through establishment of construction goal for overhaul plan of distribution grid, this article has designed an optimization system for overhaul plan of distribution grid and introduces relevant functions of the system in detail, for the purpose of settling the problems, namely repeated power failure, low work efficiency and the reasonable distribution of resources including time, manpower, materials, etc., so as to make the arrangement of overhaul plan more scientific, reliable, economic, efficient, reasonable, simple and convenient.
\end{abstract}

Keywords. overhaul plan; power equipment; overhaul strategy; safety design

\section{Introduction}

The normal operation of power equipment is important for the reliable operation of power system[1]. In its long-time running, the health condition will be worsen due to various factors. And the overhaul aims at ensuring the normal operation of power grid by extension of service life using particular measures or at least by delaying the moment of occurrence of the next failure[2,3]. However, in fact, insufficient or frequent overhaul will always occur. The insufficient overhaul may reduce the operation reliability of power grid, which results in excessive failure loss. On the contrary, the excessively frequent overhaul will ensure the reliability of power grid, which, however, would increase the overhaul costs or even reduce the equipment availability due to frequent outage from overhaul, and then lower the operation performance of the whole power grid.

For general power equipment, the traditional overhaul method uses periodic and post-failure overhaul pattern[4]. The periodic overhaul is one of the overhaul methods for power equipment based on preventive experiment. Pervious periodic overhaul regularly conducts preventive trial and maintenance to efficiently identify the potential equipment hazard, which plays a significant role in prevention of accidents and the safe operation of power grid.

This article proposes an overhaul method for distribution grid based on birth-death process of equipment, makes an analysis on the actual overhaul condition for condition-based overhaul according to basic features of condition-based overhaul, proposes a reasonable assessment model of reliability to conduct the reliability analysis, and finally optimizes the strategy for equipment overhaul. 


\section{Relevant researches}

The constant development of overhaul methods increasingly improves the productivity of equipment. The rise of assessment capability of equipment conditions requires us to positively explore a new overhaul method to adapt to the operative condition of power system. The promotion of conditionbased overhaul propels us to research a more reasonable overhaul strategy by constant summary of precedent findings and integration of their advantages based on actual equipment conditions.

The failure rate of power equipment is an important index of reliability for power equipment, generally used to measure the performance of equipment. The literature[5] introduces the bathtub curve of service life distribution for power equipment, and uses Marquardt method to predict the optimum parameters for failure rate functions, by which the division points for different life failure periods of equipment are determined. The literature[6], taking into account various factors that affect basic parameters of reliability, proposes to use least square method of curve fitting to obtain the failure rate of equipment to determine the basic parameters of reliability more accurately, which raises the reasonableness of reliability assessment for power system. The condition-based overhaul features controlling the actual equipment conditions, diagnosing and analyzing the actual situations to reasonably implement the overhaul process. The previous judgment of operation conditions for equipment is generally realized by preventive trial for power failure and regular overhaul, with relatively simple monitoring amount. The literature[7]-[10] respectively proposes condition assessment model for power transformer based on fuzzy synthetic discrimination, condition assessment technology, Bayesian network and multi-information integration. The literature[11] assesses the performance of current conditions for equipment and quantitatively analyzes the relations between equipment overhaul and system operation according to the condition monitoring results of equipment. Furthermore, it adopts generic algorithm to establish a strategic model of overhaul optimization, which minimizes the operation and overhaul costs of system. Currently, the management of whole life cycle for assets requires using the whole life cycle of equipment to manage the equipment assets to further increase the usage efficiency of equipment. The literature[12] analyzes the formulation method of condition-based overhaul strategy for electric transmission and transformation equipment in the perspective of costs of whole life cycle. The literature[13] researches the relation between overhaul cycle and equipment failure rate and analyzes the strategic decisionmaking method in different overhaul patterns based on LCC theory. The whole life cycle management can reasonably arrange the overhaul resources and formulate an optimized overhaul plan.

The equipment may age due to various factors in the life cycle, and the aging process is a random course. The equipment conditions transmit among every condition according to certain probability distribution, and the future conditions cannot be ensured. The literature[14] introduces the application of state diagram in the overhaul model. The state diagram contains the state of degradation, failure and overhaul. While the model of state diagram can be described by Markoff process model[15]. The Markoff process can well solve the probability distribution issue in aging model, so as to obtain parameters such as availability of equipment. The literature[16] uses semi-Markoff process of continuous time to describe the aging process of equipment, analyzes the connection between semiMarkoff process and aging process, and solves the steady probability of various states of system.

\section{Detection model based on Markoff model}

The state diagram is generally able to present the degradation, detection and overhaul state in the aging process model of power equipment. In Maths, the state diagram can be presented as a Markoff process. When formulating a state diagram, the relation between each state and the occurrence of transmission for system shall be considered. Furthermore, the operation law and actual conditions of system shall be profoundly controlled[16].

\subsection{Aging and failure model}


In many occasions, the system failures can be divided into two types: one is random failure, and the other system degradation (aging) result. Generally, the average MTBF and average maintenance time are assumed to be in exponential distribution. The failure probability of random failure is constant and irrelevant to equipment. The literature[2] describes aging model of equipment including random failure state and aging failure state, as shown in Figure 1. The abovementioned model describes the failure state into which the equipment going through natural wear and aging process may finally get, and the equipment will also get into random failure state FO due to accidental failure. The random failure is inevitable, and the probability where each degradation state gets into random failure state is also constant. Going through k stages of degradation, the equipment, if left unmaintained, will get into aging failure state $\mathrm{F} 1$. The post-failure maintenance will recover the equipment to $\mathrm{S} 1$ state. The rates of transfer from aging failure state F1 and random failure state F0 to a brand new state are respectively $\mu 1$ and $\mu 0$. The rate of transfer from degradative working state to random failure state is $\lambda$.

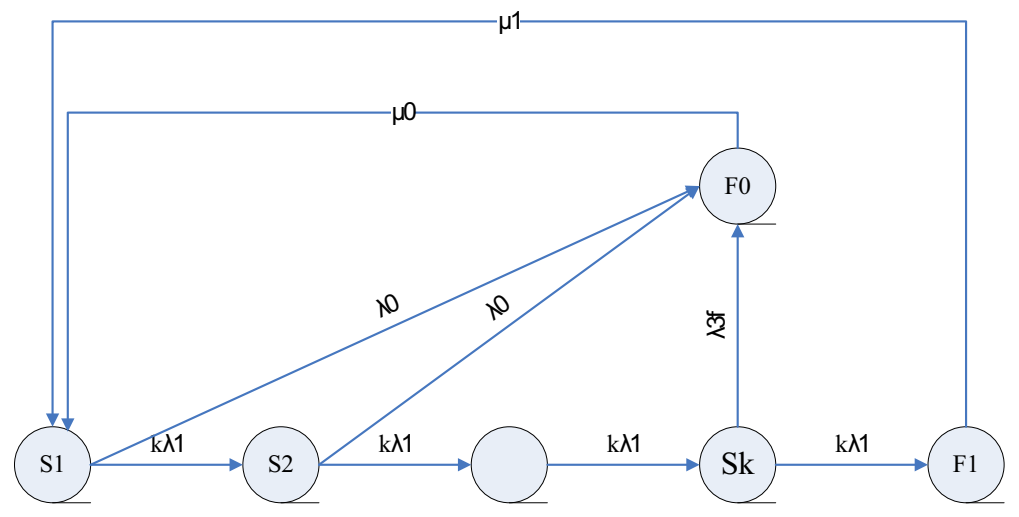

Figure 1. Diagram of degradation state containing maintenance

\subsection{Maintenance model}

Based on the abovementioned assumption, many systems will be regularly overhauled, and interfered as the equipment state reaches the critical point to avoid failure. So the implementation of maintenance can be divided into two situations, one is to proceed as preplanned, and the other to regularly monitor the system for the purpose of making sure that the overhaul and its content are being conducted. According to literature [3], the additional states M1-M3 may be introduced in overhaul process, as shown in Figure 2. In this figure, it assumes that the overhaul will generally improve the system to the previous degradation state.

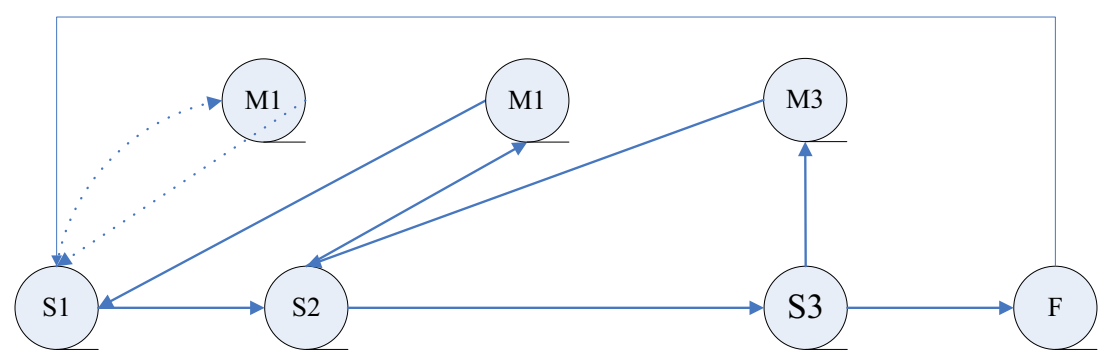

Figure 2. Model of degradation state containing maintenance

As for Figure 2, if we assume that the time of transfer among each state is in exponential distribution, the probability of its states will be constant, and the next state of system will depend on the current state. And then, what the state diagram currently presents is the Markoff process, in which the next process is irrelevant to any previous ones. 
In fact, the aging process of equipment contains not only different degradation stages and failure states, but also the monitor state, overhaul state, strategy, etc. of equipment. We assume that the equipment can be transited from degradation state and failure state to other states, and then return to one degradation or failure state (if a system failure results from overhaul). The maintenance strategies for most of the equipment are formulated through state monitoring. Furthermore, the state monitoring is applied more widely in actual conditions, therefore the introduction of state check in the model is very essential.

\section{Analysis of reliability and example}

In the Markoff process model, the system going through $\mathrm{n}$ steps and transferred from one state to another will get into limit state, namely steady state. The probability of steady state after entering the steady state is a constant, which is irrelevant to the original state. When the state number is limited, the steady probability is generally existent. We can consider the system is in steady state, and each of its state probability will no longer change even the system goes through one more transfer process.

\subsection{Reliability analysis}

When in steady state, the average time for which the system remains in state i per unit time is called frequency in state $i$, namely fi. The duration of state $i$ refers to the average duration that the system remains in state $i$ when it is in steady state. The average time of not being in state $f$ and the time of not being in state $\mathrm{f}$ for system are respectively $\mathrm{Ti}$ and $\mathrm{Ti}$ ', so the average cycle time is:

$$
T_{c i}=T_{i}+T_{i}^{\prime}
$$

Known from the definition of frequency, the frequency fi equals to reciprocal of average cycle time for a long period of time, namely:

$$
f_{i}=\frac{1}{T_{c i}}
$$

Multiply two sides by $\mathrm{Ti}$, and we get:

$$
f_{i} T_{i}=\frac{T_{i}}{T_{c i}}
$$

The state probability of being in state i for a long period of time is $\frac{T_{i}}{T_{c i}}$, namely:

$$
\frac{T_{i}}{T_{c i}}=p_{i}
$$

So:

$$
f_{i}=\frac{1}{T_{c i}}=\frac{T_{i}}{T_{c i}} \times \frac{1}{T_{i}}=\frac{p_{i}}{T_{i}}
$$

Then we focus on the relation among state frequency $f_{i}$, state duration $T_{f}$ and transfer rate of each state in system. Firstly, the transfer frequency $f_{i j}$ is defined as the expected frequency of direct transfer from state $\mathrm{i}$ to state $\mathrm{j}$, which may be presented as:

$$
\begin{aligned}
f_{i j} & =\lim _{\square \rightarrow 0} \frac{1}{\square t} p\{[X(t+\square t)=j \mid X(t)=i]\} \\
& =\lim _{\square \rightarrow 0} \frac{1}{\square t} p\{[X(t+\square t)=j \mid X(t)=i]\} p[X(t)=i] \\
& =\lim _{\square t \rightarrow 0} \frac{1}{\square t}\left(\lambda_{i j} \square t\right) p[X(t)=i]=\lambda_{i j} p_{i}
\end{aligned}
$$


Therefore, the transfer frequency is a conditional probability based on the system being in state $\mathrm{i}$ in $t$ moment. According to the definition, the frequency in state $\mathrm{i}$ shall be the sum of all frequencies of transfer from state i, namely:

$$
f_{i}=\sum_{i \neq j} f_{i j}
$$

Substitute it in formula 6 , and we get:

$$
f_{i}=\sum_{i \neq j} f_{i j}=p_{i} \sum_{j \neq i} \lambda_{i j}=\sum_{j \neq i} p_{j} \lambda_{j i}
$$

When becoming steady, the sum of frequencies of transfer from other states to state $\mathrm{i}$ for system is equal to the sum of frequencies of transfer from state $i$, namely the frequency balance. So the duration $T_{i}$ of being in state i may be presented as:

$$
T_{i}=\frac{p_{i}}{f_{i}}=\frac{1}{\sum_{i \neq j} \lambda_{i j}}
$$

\subsection{Example analysis}

Take the data from literature [2] for example to calculate the steady state probability of circuit breaker and average duration and access frequency for each state. The rate of transfer among each state is shown in Table 1.

Table 1. Value of state transfer rate

\begin{tabular}{cccc}
\hline Transfer rate & Value(1/year) & Transfer rate & Value(1/year) \\
\hline$\lambda_{1}$ & 0.33 & $\delta_{1}$ & 360 \\
$\lambda_{2}$ & 0.29 & $\delta_{2}$ & 360 \\
$\lambda_{3}$ & 0.5 & $\delta_{3}$ & 360 \\
$\gamma_{1}$ & 0.5 & $\mu_{1}$ & 12 \\
$\gamma_{2}$ & 1 & $\mu_{2}$ & 360 \\
$\gamma_{3}$ & 1 & $\mu_{3}$ & 180 \\
\hline
\end{tabular}

Set the state probabilities of S1、S2、S3、F、I1、I2、I3 and M3 respectively to: P0、P1、 $\mathrm{P} 2 、 \mathrm{P} 3 、 \mathrm{P} 4 、 \mathrm{P} 5 、 \mathrm{P} 6 、 \mathrm{P} 7 、 \mathrm{P} 8$, and establish the state transfer matrix:

$$
P(n)=\left[\begin{array}{ccccccccc}
1-\lambda_{1}-\gamma_{1} & \lambda_{1} & 0 & 0 & \gamma_{1} & 0 & 0 & 0 & 0 \\
0 & 1-\lambda_{2}-\gamma_{2} & \lambda_{2} & 0 & 0 & \gamma_{2} & 0 & 0 & 0 \\
0 & 0 & 1-\lambda_{3}-\gamma_{3} & \lambda_{3} & 0 & 0 & 0 & \gamma_{3} & 0 \\
\mu_{F} & 0 & 0 & 1-\mu_{F} & 0 & 0 & 0 & 0 & 0 \\
\delta_{1} & 0 & 0 & 0 & 1-\delta_{1} & 0 & 0 & 0 & 0 \\
0 & 0 & 0 & 0 & 0 & 1-\delta_{2} & \delta_{2} & 0 & 0 \\
\mu_{2} & 0 & 0 & 0 & 0 & 0 & 1-\mu_{2} & 0 & 0 \\
0 & 0 & 0 & 0 & 0 & 0 & 0 & 1-\delta_{3} & \delta_{3} \\
0 & \mu_{3} & 0 & 0 & 0 & 0 & 0 & 0 & 1-\mu_{3}
\end{array}\right]
$$

Therefore we get: 


$$
P(n)=\left[\begin{array}{c}
P_{0} \\
P_{1} \\
P_{2} \\
P_{3} \\
P_{4} \\
P_{5} \\
P_{6} \\
P_{7} \\
P_{8}
\end{array}\right]\left[\begin{array}{ccccccccc}
1 & 1 & 1 & 1 & 1 & 1 & 1 & 1 & 1 \\
\lambda_{1} & -\lambda_{2}-\gamma_{2} & \lambda_{2} & 0 & 0 & 0 & 0 & 0 & \mu_{3} \\
0 & \lambda_{2} & -\lambda_{3}-\gamma_{3} & 0 & 0 & 0 & 0 & \gamma_{3} & 0 \\
0 & 0 & \lambda_{3} & -\mu_{F} & 0 & 0 & 0 & 0 & 0 \\
\gamma_{1} & 0 & 0 & 0 & -\delta_{1} & 0 & 0 & 0 & 0 \\
0 & \gamma_{2} & 0 & 0 & 0 & -\delta_{2} & 0 & 0 & 0 \\
0 & 0 & 0 & 0 & 0 & \delta_{2} & -\mu_{2} & 0 & 0 \\
0 & 0 & \gamma_{3} & 0 & 0 & 0 & 0 & -\delta_{3} & 0 \\
0 & 0 & 0 & 0 & 0 & 0 & 0 & \delta_{3} & -\mu_{3}
\end{array}\right]^{-1}\left[\begin{array}{l}
1 \\
0 \\
0 \\
0 \\
0 \\
0 \\
0 \\
0 \\
0
\end{array}\right]
$$

Substitute the data, and we get:

$$
P(n)=\left[\begin{array}{c}
P_{0} \\
P_{1} \\
P_{2} \\
P_{3} \\
P_{4} \\
P_{5} \\
P_{6} \\
P_{7} \\
P_{8}
\end{array}\right]=\left[\begin{array}{l}
0.7326 \\
0.2204 \\
0.0426 \\
0.0018 \\
0.0010 \\
0.0006 \\
0.0006 \\
0.0001 \\
0.0002
\end{array}\right]
$$

According to formula 7 and formula 8, we can get:

$$
\begin{aligned}
& f_{s_{1}}=p_{0}\left(\lambda_{1}+\gamma_{1}\right)=0.7326(0.33+0.5)=0.608 \\
& f_{s 2}=p_{1}\left(\lambda_{2}+\gamma_{2}\right)=0.2204(0.29+0.1)=0.284 \\
& f_{s_{3}}=p_{2}\left(\lambda_{3}+\gamma_{3}\right)=0.0426(0.5+0.1)=0.0639 \\
& f_{F}=p_{3} \mu_{F}=0.0018 \times 12=0.0216 \\
& T_{s 1}=\frac{1}{\lambda_{1}+\gamma_{1}}=\frac{1}{0.33+0.5}=1.205 \\
& T_{s 2}=\frac{1}{\lambda_{2}+\gamma_{2}}=\frac{1}{0.29+1}=0.775 \\
& T_{s 3}=\frac{1}{\lambda_{3}+\gamma_{3}}=\frac{1}{0.29+1}=0.667 \\
& T_{F}=\frac{1}{\mu_{F}}=\frac{1}{12}=0.083
\end{aligned}
$$


This article has researched and developed optimization system for overhaul plan of distribution grid based on the features and actual demand of distribution grid overhaul and dependent on the control integration project of Yizhou Power Supply Company, Shanxi Power Company, State Grid. It adequately takes into account the voltage class and state of equipment, and realizes the scientific reasonableness of overhaul by method of overhaul classification. The optimization system for overhaul plan of distribution grid is a complicated project involving an extremely wide range. It can proceed to be researched in the following aspects: Deeply research the accuracy and reliability of state assessment for power equipment to avoid blind qualitative from personal experience; The GPIS positioning system can be taken into account to accurately position the geographical information of power equipment to facilitate the power overhaul.

\section{References}

1. Guo Yongji. Principle and Application of Power System Reliability[M]. Beijing: Tsinghua University Press, 1986.

2. Endrenyi J, Anders G, Leite da Silva. A Probabilistic Evaluation of the Effect of Maintenance on Reliability - An Application[J]. IEEE Transactions on Power Systems, 1998, 13(2):576-583.

3. Endrenyi J, Aboresheid S, Allan R, et a1. The Present Status of Maintenance Strategies and the Impact of Maintenance on Reliability[J]. IEEE Transactions on Power Systems, 2001, 16(4):638646.

4. Sun Huaidong, Lu Yichun. Reflection on Overhaul of Implementation Status of Power Generating Equipment[J]. Huadong Power, 2002:37-39.

5. Zhang Li, Zhang Bo. Optimum Estimate Method of Failure Rate Parameters for Electrical Equipment[J]. Relay, 2005, 33(17):31-34.

6. Han $\mathrm{Wu}$, Liu Zongbing, Liu Juan, Wang Lin. Obtention of Equipment Failure Rate from Least Square Method of Curve Fitting[J]. Yunnan Power Technology, 2008, 36(5):5-7.

7. Liao Ruijin, Wang Qian, Luo Sijia, Liao Yuxiang, Sun Caixin. Assessment Model of Operation State of Power Transformer Based on Fuzzy Synthetic Discrimination[J]. Automation of Electric Power Systems, 2008, 32(3):70-75.

8. Lu Zhihao, Huang Hua, Fu Chenzhao. Preliminary Exploration of Life Assessment for Power Transformer Based on State Assessment Technology[J]. 2009, 37(6):1019-1022.

9. Zhao Wenqing, Zhu Yongli, Jiang Bo, Zhang Xiaoqi, Zhang Bin. State Assessment of Power Transformer Based on Bayesian Network[J]. High Voltage Technology, 2008, 34(5):1032-1039.

10. Liao Ruijin, Huang Feilong, Yang Lijun, Zheng Hanbo, Gong Jing. State Assessment Model of multi-information integrated Power Transformer[J]. 2010, 36(6):1455-1460.

11. YANG Z M, Djurdjanovic D, NI J. Maintenance scheduling in manufacturing systems based on predicted machine degradation[J]. Journal of Intelligent Manufacturing, 2008, 19(1):87-98.

12. Wang Jiaming, Liu Wenying, Wei Fan, et al.. Overhaul Strategy Research of Electric Transmission and Transformation Equipment State Based on Costs Management of Life Cycle[J]. Power System Protection and Control, 2011, 39(5):77-80.

13. Ma Zhongneng, Zhong Lihua, Lu Kai. Optimum Overhaul Strategy Research Based on Whole Life Cycle of Power Equipment [J]. Power System Protection and Control, 2011, 39(16): 34-39.

14. Thomas M. Welte. Using State Diagrams for Modeling Maintenance of Deteriorating Systems[J]. IEEE Trans. Power Systems, 2009(1):58-66.

15. S. Ross. Stochastic Processes[M]. Pearson Education, Inc. 2nd edition, 1996.

16. Curtis L, Tomasevicz, Sohrab Asgarpoor. Preventive Maintenance Using Continuous-Time SemiMarkov Processes[C]. Proceedings of the 3 8th North American Power Symposium, 2006, 09:38. 